\title{
Evagination of metacestodes of the WFU strain of Taenia crassiceps and evaluation of the impact of immune suppression of hamsters during tapeworm development
}

\author{
Laura Aguilar-Vega ${ }^{1}$, Tanya Plett-Torres ${ }^{2}$, Marta C. Romano $^{3}$ and Rimma Zurabian ${ }^{1}$ \\ ${ }^{1}$ Departamento de Microbiología y Parasitología, Facultad de Medicina, Universidad Nacional Autónoma de México, Ciudad de \\ México, Mexico; \\ ${ }_{2}^{2}$ Plan de Estudios Combinados en Medicina (PECEM), Facultad de Medicina, Universidad Nacional Autónoma de México, Ciudad \\ de México, Mexico; \\ ${ }^{3}$ Departamento de Fisiología, Biofísica y Neurociencias, CINVESTAV del I.P.N., Ciudad de México, Mexico
}

\begin{abstract}
Taeniosis-cysticercosis caused by Taenia crassiceps (Zeder, 1800) is a useful experimental model for biomedical research, in substitution of Taenia solium Linnaeus, 1758, studied during decades to develop effective vaccination, novel anti-helminthic drugs and diagnostic tools. Cysticercosis in mouse (Mus musculus Linnaeus) is achieved by the larval subculturing of the Wake Forest University (WFU) strain of T. crassiceps. Golden hamster, Mesocricetus auratus (Waterhouse), has been shown to be the most suitable host for adult forms of parasite in experimental taeniosis. Metacestodes of $T$. crassiceps WFU multiply by budding without restrictions once inoculated into the mouse, while the number of tapeworms developed from these larvae in hamsters remains highly variable. Three objectives have been proposed to improve the infection of T. crassiceps WFU in hamsters: (1) to re-evaluate the need of immune suppression; (2) to investigate the advantage of infecting hamsters with metacestodes with in vitro protruded scolices; and (3) to compare a number of tapeworms developed from metacestodes subcultured in hamsters against those proliferated in mice. Our results demonstrated that when the evagination of murine metacestodes was high, the number of $T$. crassiceps WFU adults obtained from hamsters was also high. Immunosuppressive treatment remains relevant for this experimental rodent model. The hamster-to-hamster cysticercosis-taeniosis by $T$. crassiceps overcame the mouse-to-hamster model in the yield of adult specimens. In vitro scolex evagination and metacestode asexual proliferation in hamsters place this rodent model by $T$. crassiceps WFU as the most affordable experimental models with taeniids.
\end{abstract}

Keywords: Parasite, Taeniidae, larvae, tapeworms, rodent cysticercosis-taeniosis

Taenia crassiceps (Zeder, 1800) is a taeniid cestode parasitising wildlife vertebrates, with three development stages: eggs, metacestodes and adults. Metacestodes or cysticerci are found in natural infections in rodents (Freeman 1962), but also in accidentally infected humans (Lescano and Zunt 2013). Two main laboratory strains of $T$. crassiceps are ORF metacestodes that have lost the scolices and the Wake Forrest University (WFU) strain, with an apparently normal larval phenotype (Everhart et al. 2004). Similar to the ORF, the WFU strain can multiply asexually in the peritoneum of mice (Mus musculus Linnaeus), but unlike the ORF strain it grows to the adult stage in hamsters as definitive hosts (Willms and Zurabian 2010).

Verster (1971) was the first to investigate immune suppression of unnatural definitive hosts, infected with taeniid parasites. In order to improve the yield of adult worms and to obtain sexually mature specimens with infective eggs, Sato and Kamiya (1989) treated hamsters infected with T. crassiceps with corticosteroids. The average recovery of $T$. crassiceps from immunosuppressed hamsters varied between 11.7 and 76.7\% (Sato et al. 1993, Zurabian et al. 2008). However, only Kitaoka et al. (1990) found eggs of T. crassiceps expelled in the faeces of hamsters, with and without steroid treatment.

Steroids were also used in the experimental taeniosis with Taenia solium Linnaeus, 1758 or Taenia pisiformis (Bloch, 1780) (Allan et al. 1991, Maravilla et al. 1998). The definitive hosts were treated with $\geq 5 \mathrm{mg} / \mathrm{kg}$ of glucocorticoids, in a single or multiple applications, resulting in a highly variable number of recovered tapeworms with different degree of sexual maturity (Sato and Kamiya 1989, 1990, Kitaoka et al. 1990, Sato et al. 1994, Avila et 

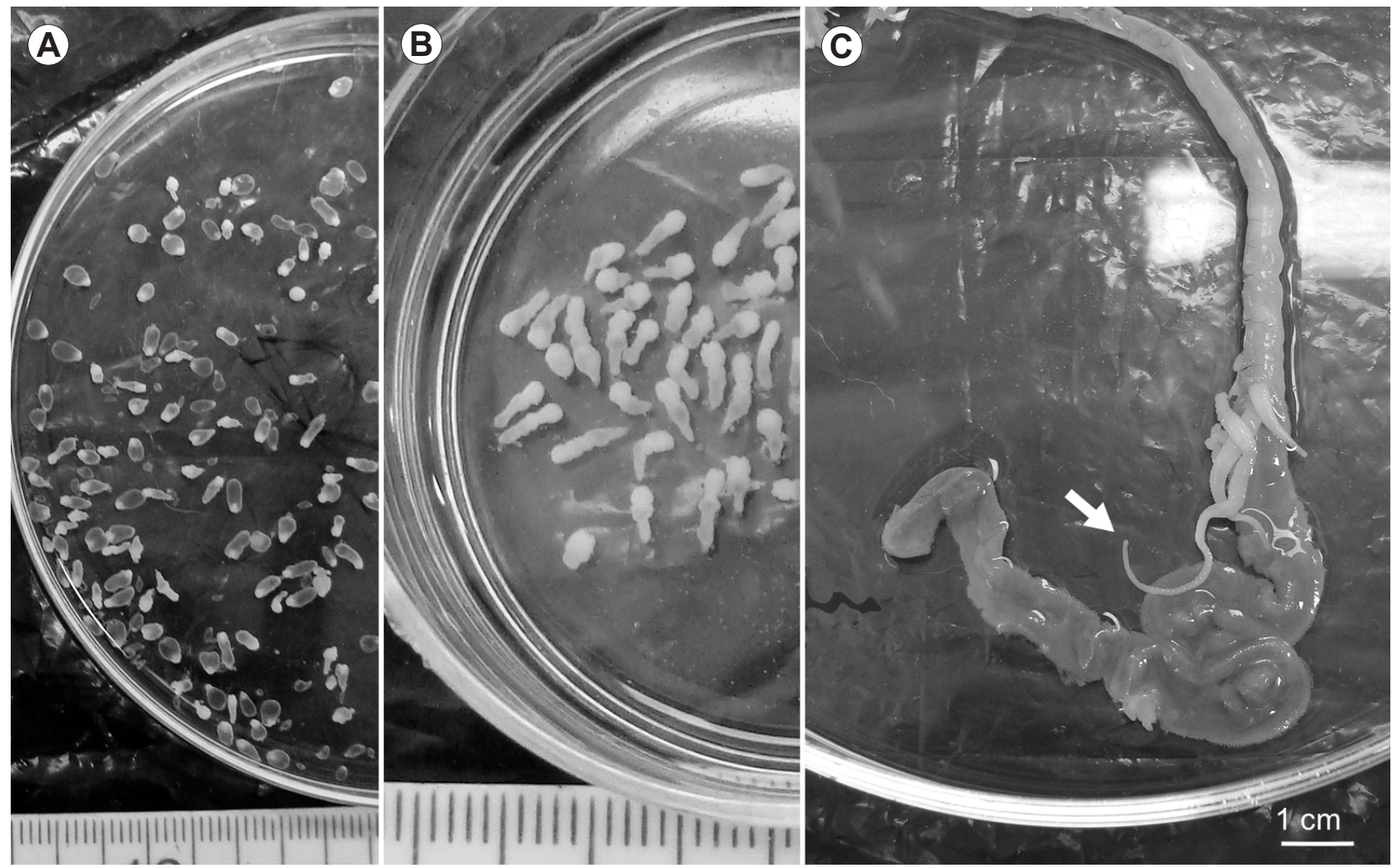

Fig. 1. Taenia crassiceps (Zeder, 1800); metacestodes and adults of the WFU strain. A - heterogeneous population of metacestodes with different degree of development obtained from mouse peritoneum; $\mathbf{B}$ - larvae with elongated scolex after in vitro incubation; C - adult tapeworms localised in the small intestine of infected hamster and detached scolex (arrow).

al. 2006), and also inducing a cachexic state in the animals (Braun et al. 2014, Archer-Lahlou et al. 2018).

The present study was pursuing three objectives related to the experimental taeniosis: (1) to re-evaluate the importance of immune suppression in order to avoid cachexic state of the hosts; (2) to investigate the advantage of infecting hamsters with metacestodes with in vitro protruded scolices; and (3) to evaluate efficiency of model and infectivity of metacestodes proliferated either in mice or hamsters as intermediate hosts.

\section{MATERIALS AND METHODS}

Proliferation of metacestodes of Taenia crassiceps in rodent hosts Metacestodes (Cs) of the WFU strain of T. crassiceps were isolated following Everhart et al. (2004). Parasites were maintained in mice by intraperitoneal (i.p.) passages as described previously (Zurabian et al. 2013). Female four weeks old BALB/c mice $(n=9)$ and female five months old golden hamsters $(n=2)$ each were inoculated with ten Cs. In total, nine consecutive infrapopulations were obtained from mice (CsM) and two infrapopulations from hamster's peritoneum $(\mathrm{CsH})$.

Animals were supplied with commercial pellets and water $a d$ libitum and kept under controlled conditions. Necropsies consisted of cervical dislocation of mice or injection of $400 \mathrm{mg} / \mathrm{kg}$ of sodium pentobarbital (Pisabental, Pisa Agropecuaria, Hidalgo, Mexico) in hamsters, as established by Mexican Official Regulation (NOM-062-ZOO-1999). The Ethical Committee of Faculty of Medicine (UNAM) had approved the experimental assays.
Criteria for selection of metacestodes and in vitro evagination

For in vitro culturing, 50-70 CsM or $\mathrm{CsH}$ with 3-4 mm large bladder and invaginated worms approximately $1 \mathrm{~mm}$ long were selected and evaluated for the contractile movements under the light microscopy. Cysticerci were washed in PBS and incubated in $10 \mathrm{mM}$ glucose in RPMI 1640 medium (Sigma-Aldrich Co., St. Louis, Missouri, USA) during $1.5 \mathrm{~h}$ at $37^{\circ} \mathrm{C}$ and $5 \% \mathrm{CO}_{2}$. After incubation, evaginated $\mathrm{Cs}$ were counted, randomly collected and fed to the hamsters (Fig. 1B). Evagination of Cs was expressed in $\%$. Only parasites with scolices irreversibly protruded out of the bladder were counted and grouped as $1-50 \%$ or $51-100 \%$. Metacestodes with inverted scolex were selected from the same infrapopulation upon necropsies and used as a control group $(0 \%$ of evagination)

\section{Adults of Taenia crassiceps in hamsters}

Female 5-6 months old hamsters were treated with $20 \mathrm{mg}$ of metronidazole (Degort's Chemical, Ciduad de México, México) per $\mathrm{kg}$ of weight given each day for three days. Albendazole (Zentel, Glaxo Smith Kline, Ciudad de México, México) was given in a dose of $30 \mathrm{mg}$ per kg of hamster's weight per day during five days. Hamsters were divided into two groups for the infection assays: animals in the group 1 had immunosuppressive treatment (ISP) with $2 \mathrm{mg} / \mathrm{kg}$ of methyl prednisolone acetate (Upjohn, Ciudad de México, Mexico) at the same day of infection, and group 2 were animals without steroid treatment (nISP).

ISP $(n=62)$ and $n I S P(n=37)$ hamsters were fed with CsM, and submitted to the evagination assay. All CsM used for assays were part of nine mouse infrapopulations studied during five-year-peri- 
Table 1. Overall distribution of the WFU strain of Taenia crassiceps (Zeder, 1800) developed from metacestodes proliferated in mice (CsM) according to the evagination rate. CsM fed to the hamsters with (ISP) and without (nISP) steroid treatment. KWT, Kruskall-Wallis test; FET, Fisher's Exact test.

\begin{tabular}{|c|c|c|c|c|c|c|c|c|}
\hline \multirow{3}{*}{$\begin{array}{l}\% \text { of evaginated } \\
\text { CsM fed to } \\
\text { the hamsters } \\
(n=99)\end{array}$} & \multirow{2}{*}{\multicolumn{2}{|c|}{$\begin{array}{l}\text { Median of tapeworms } \\
(\mathrm{p} 25, \mathrm{p} 75)\end{array}$}} & \multicolumn{6}{|c|}{ Infection by at least 1 tapeworm } \\
\hline & & & \multirow{2}{*}{$\begin{array}{l}\mathrm{KWT} \\
p \text { value }\end{array}$} & \multicolumn{2}{|c|}{$\mathrm{nISP} \mathrm{n}=37$} & \multicolumn{2}{|c|}{ ISP $n=62$} & \multirow{2}{*}{$\begin{array}{c}\text { FET } \\
p \text { value }\end{array}$} \\
\hline & nISP $\mathrm{n}=37$ & $\mathrm{ISP} n=62$ & & Negative $=33$ & Positive $=4$ & Negative $=18$ & Positive $=44$ & \\
\hline $0(25)$ & $0(0,0)$ & $0.5(0,4)$ & 0.0516 & $9(27)$ & $2(50)$ & $7(39)$ & $7(16)$ & 0.208 \\
\hline $1-50(24)$ & $0(0,0)^{\mathrm{a}}$ & $1(0,2)$ & 0.0171 & $10(30)$ & $1(25)$ & $6(33)$ & $7(16)$ & 0.033 \\
\hline $51-100(50)$ & $0(0,0)^{\mathrm{b}}$ & $3(1,4)$ & 0.0001 & $14(42)$ & $1(25)$ & $5(28)$ & $30(68)$ & $<0.001$ \\
\hline
\end{tabular}

Table 2. Overall distribution of the WFU strain of Taenia crassiceps (Zeder, 1800) developed from metacestodes proliferated in hamster $(\mathrm{CsH})$ according to the evagination rate. $\mathrm{CsH}$ were fed to the non-treated with steroids hosts. KWT, Kruskall-Wallis test; FET, Fisher's Exact test.

\begin{tabular}{|c|c|c|c|c|c|}
\hline \multirow{2}{*}{$\begin{array}{l}\% \text { of evaginated } \mathrm{CsH} \text { fed } \\
\text { to the hamsters }(\mathrm{n}=12)\end{array}$} & \multirow{2}{*}{$\begin{array}{l}\text { Median of tapeworms } \\
(\mathrm{p} 25, \mathrm{p} 75)\end{array}$} & \multirow{2}{*}{$\begin{array}{c}\text { KWT } \\
p \text { value }\end{array}$} & \multicolumn{2}{|c|}{ Infection by at least 1 tapeworm } & \multirow{2}{*}{$\begin{array}{c}\text { FET } \\
p \text { value }\end{array}$} \\
\hline & & & Negative $n=5$ & Positive $\mathrm{n}=7$ & \\
\hline $0(4)$ & $3.5(2.5,4)$ & & $0(0)$ & $4(57)$ & \\
\hline $1-50(4)$ & $2(0.5,4.5)$ & 0.033 & $1(20)$ & $3(43)$ & 0.030 \\
\hline $51-100(4)$ & $0(0,0)$ & & $4(80)$ & $0(0)$ & \\
\hline
\end{tabular}

od. Twelve nISP hamsters were fed with evaginated CsH selected from two infrapopulations reared in the hamster's peritoneum. In infections with CsM, each experimental group of hamsters consisted of five to seven animals, and $\mathrm{CsH}$ were used to infect groups of four animals.

The infectivity was calculated using the next formula: (number of hosts infected with parasites $\times 100$ ) / total number of hosts. The efficiency of infections was calculated using the following equation: (number of tapeworms $\times 100$ ) / (number of hosts $\times 10$ metacestodes).

Adults of $T$. crassiceps WFU specimens were obtained from the hamsters' gut after 1-2 weeks of growth, counted and measured. The largest tapeworm was fixed and stained following Paracarmin-Mayer method (Lamothe-Argumedo 1997) in order to evaluate the parasite's stage of development.

\section{Statistical analysis}

Two statistical tests were used to analyse the data related to the experimental taeniosis. The Kruskal-Wallis test (KWT) was used to evaluate the number of tapeworms obtained from each group of hamsters, and the Fisher Exact test (FET) was used to analyse the difference between the frequencies of hosts harbouring at least one tapeworm. The results obtained after infection of ISP or nISP hamsters with CsM or $\mathrm{CsH}$ with different evagination percentages $(0,1-50$ or $51-100 \%)$ were compared using KWT or FET. Significant differences were considered when $\mathrm{p}<0.05$.

\section{RESULTS}

\section{Evagination of metacestodes of the WFU strain of Taenia crassiceps}

Each CsM or $\mathrm{CsH}$ infrapopulation remained in the rodent peritoneum for 5-6 months and comprised Cs with different degree of development (Fig. 1A). Selected for the in vitro assay $\mathrm{CsM}$ or $\mathrm{CsH}$ had apparently similar phenotypes and size, but displayed different evagination rates during incubation.

\section{Adults of Taenia crassiceps WFU developed from CsM}

Hamsters developed a significantly higher number of adults when infected with CsM from the 51-100\% evagination group, compared to those with a lower evagination number or control ( $p=0.026$, KWT; data not shown in tables). Similarly, when greater evagination was achieved, the greater median was obtained for tapeworms from ISP hamsters (Table 1). More hamsters lodged at least one adult specimen when fed with CsM with $51-100 \%$ of evagination ( $p=0.024$, FET; data not in tables); the highest frequency of infection was also seen in ISP hosts $(68 \%$, $p<0.001$, FET; Table 1).

\section{Adults of Taenia crassiceps WFU developed from CsH}

Control metacestodes (without evagination) produced a major number of adults per host $(\mathrm{p}=0.033$, KWT; Table 2). All hamsters fed with $\mathrm{CsH}$ without evagination harboured at least one adult specimen.

\section{Taenia crassiceps WFU tapeworms in hamsters}

Infectivity and efficiency was calculated for total populations of hamsters $(n=111)$ infected (during five-years period) either with $\mathrm{CsM}$ or $\mathrm{CsH}$ (Table 3). Tapeworms were found in $58 \%$ and $49 \%$ of hamsters infected with $\mathrm{CsH}$ or CsM, respectively. Infectivity of CsH (19\%) was higher than CsM (15\%) as shown in Table 3.

The number of tapeworms per host varied between one and nine, and $50 \%$ of the total number of hamsters used in the study had at least one intestinal tapeworm upon necropsy. The highest number of $T$. crassiceps WFU tapeworms was found attached to the intestinal wall of ISP hamsters. The largest tapeworms were also found in ISP hamsters, and measured about $15 \mathrm{~cm}$ in unrelaxed state (Fig. 1C). Staining with Mayer's paracarmin revealed pregravid proglottids and eggs in the process of formation. 
Table 3. Efficiency of taeniosis model and infectivity of Taenia crassiceps WFU metacestodes. CsM, cysticerci proliferated in mice; $\mathrm{CsH}$, cysticerci proliferated in hamsters. Efficiency = (number of hosts infected with parasites $\times 100$ ) / total number of hosts. Infectivity $=($ number of tapeworms $\times 100) /$ (number of hosts $\times 10$ metacestodes).

\begin{tabular}{lccc}
\hline $\begin{array}{l}\text { No. of the hamsters fed } \\
\text { with metacestodes }\end{array}$ & Tapeworms (n) Efficiency (\%) Infectivity (\%) \\
\hline $\mathrm{CsM} \mathrm{n}=99$ & 149 & 49 & 15 \\
$\mathrm{CsH} \mathrm{n}=12$ & 23 & 58 & 19 \\
\hline
\end{tabular}

\section{DISCUSSION}

Metacestodes of the WFU strain of Taenia crassiceps used in this study descended from i.p. passages of mice maintained during almost one decade; data for this study were collected from nine consecutive Cs infrapopulations. As previously shown, this parasite loses the infectivity in experimental definitive hosts (hamsters) after long periods of asexual sub-culturing in mice, but it can be reactivated after parasite's natural life cycle (Zurabian et al. 2008).

Our results revealed that CsM with high evagination rates were able to continue with further development and, the number of tapeworms developed from these metacestodes, was significantly higher than the number of adults from its non-evaginated counterparts. We assume that because of the extra glucose administration, activated scolices might have an enhanced ability to anchor to the intestinal wall of the host. The cysticerci exposed to high glucose concentration might have an improved metabolism that, besides other developmental effects, induced a faster growth or differentiation of the hooks.

The presence of a sodium-dependent glucose transporter has been shown in larval and adult Taenia solium (see Cornford et al. 2001) and the parasite expresses enzymes of the pentose cycle (Rendón et al. 2008). It is well known that treatment of cysticerci with cysticide albendazole selectively inhibits the uptake of glucose, leading to glycogen storage depletion in the parasite (Vinaud et al. 2008).

We found that hamsters infected with evaginated WFU murine cysticerci (CsM) and a single dose of methyl prednisolone acetate developed a significantly higher number of worms, confirming that the immune suppression is important for the tapeworm development in the host. Therefore, our efforts to avoid immune suppression were not successful in mouse-to-hamster infections. Better results were obtained when asexually proliferated $\mathrm{CsH}$ were fed to hamsters non-treated with steroids, ending up in the taeniosis model with higher efficiency. Hence, the growth of cysticerci in hamsters permits to exclude the host immunosuppression.

Similar to reports of experimental taeniosis caused by Taenia pisiformis or T. solium in immunosuppressed hamsters (Maravilla et al. 1998, Toral-Bastida et al. 2011, Domínguez-Roldan et al. 2016), we observed that almost half of the studied hamsters became infected with $T$. crassiceps WFU. It should be pointed out that cysticerci used in these studies were resected from their natural hosts (rabbit and pig), had a larger scolex and fixative structures when compared to the smaller ones in WFU larvae (Beveridge and Pickard 1976, Loos-Frank 2000). Efficiency of our model can be considered high enough, despite of the reduced infectivity over years of asexual proliferation (Zurabian et al. 2008), and the presence of morphological aberrations related to the hooks or suckers (Aguilar-Vega et al. 2016).

Regarding infectivity, compared to the above mentioned studies that gave 3-6 taeniid larvae to each definitive host, we administered ten WFU metacestodes, a strategy that prevented small worms from being swept away by peristalsis (Freeman et al. 2011, Pospekhova and Bondarenko 2014), and promoting a high load of helminths in the small intestine of the host. Upon necropsy, we did not search for detached complete tapeworms or gravid proglottids in the hamsters' faeces, and counted only those embedded in the intestinal mucosa.

For the first time, T. crassiceps WFU metacestodes were inoculated into the peritoneum of hamsters $(\mathrm{CsH})$ and, after asexual multiplication, were evaginated and orally fed to another hamster. Only non-steroid treated hamsters were used for the assay, and the results suggest that better yields concerning the number of tapeworms were achieved when infection was done using non-evaginated metacestodes. As contradictory as it may seem, the greater the evagination of CsM, there was a greater infectivity.

In summary, a higher infectivity and efficiency were achieved in the new experimental model of taeniosis produced by T. crassiceps WFU cysticerci proliferated in hamsters compared to the mouse-based taeniosis model. The hamster-to-hamster model avoids the immune suppression and additional cysticerci manipulations before being administrated to the definitive host. More studies should be done to set hamsters as the best hosts for larval and adult stages of $T$. crassiceps WFU.

Acknowledgements. Financial support was provided by the División de Investigación, Facultad de Medicina, UNAM. Thanks are due to Ulises Nares Galindo for support in extraction of adult parasites.

\section{REFERENCES}

Aguilar-Vega L., García-Prieto L., Zurabian R. 2016: Abnormalities in the WFU strain of Taenia crassiceps (Cyclophyllidea: Taeniidae) following years of propagation in mice. J. Helminthol. 90: 1-6.
Allan J.C., Garcia-Dominguez C., Craig P.S., Rogan M.T., Lowe B.S., Flisser A. 1991: Sexual development of Taenia solium in hamsters. Ann. Trop. Med. Parasitol. 85: 573-576.

Archer-Lahlou E., Lan C., Jagoe R.T. 2018: Physiological culture conditions alter myotube morphology and responses to 
atrophy treatments: implications for in vitro research on muscle wasting. Physiol. Rep. 6: e13726.

Avila G., Teran N., Aguilar-Vega L., Maravilla P., Mata-Miranda P., Flisser A. 2006: Laboratory animal models for human Taenia solium. Parasitol. Int. 55: S99-S103.

Beveridge I., Rickard M.D. 1976: The development of the rostellar hooks of Taenia pisiformis. Int. J. Parasitol. 6: 55-59.

Braun T.P., Szumowski M., Levasseur P.R., Grossberg A.J., Zhu X., Agarwal A., Marks D.L. 2014: Muscle atrophy in response to cytotoxic chemotherapy is dependent on intact glucocorticoid signalling in skeletal muscle. PLoS ONE 9: e106489.

Cornford E.M., Cornford M.E, Wright E.M., Bruckner D.A., Sampogna S., Hirayama B.A. 2001: Human cerebral cysticercosis: immunolocalization of a sodium-dependent glucose cotransporter (SGLT) in larval and adult tapeworms. J. Parasitol. 87: 510-521.

Domínguez-Roldan R., Hallal-Calleros C., Scuitto E., Hernández M., Aguirre-Flores V., García-Jiménez S., Báez-Saldaña A., Flores-Pérez F.I. 2016: Behavioural and hormonal changes associated with the infective dose in experimental taeniasis in golden hamsters (Mesocricetus auratus). Exp. Parasitol. 166: 173-180.

Everhart M.E., Kuhn R.E., Zelmer D.A. 2004: Infrapopulation dynamics of a wild strain of Taenia crassiceps (WFU) (Cestoda: Taeniidae) in BALB/cJ mice. J. Parasitol. 90: 79-84.

Freeman R.S. 1962: Studies on the biology of Taenia crassiceps (Zeder, 1800) Rudolphi, 1810 (Cestoda). Can. J. Zool. 40: 969990.

Freeman R.S., Adorjan A., Pimlott D.H. 2011: Cestodes of wolves, coyotes, and coyote-dog hybrids in Ontario. Can. J. Zool. 39: 527-532.

Kiтаока M., Окu Y., Окамото M., Kамiya M. 1990: Development and sexual maturation of Taenia crassiceps (Cestoda) in the golden hamster. J. Parasitol. 76: 399-402.

LAmothe-Argumedo R. 1997: Manual de técnicas para preparar y estudiar parásitos de animales silvestres. AGT, México, D.F., $43 \mathrm{pp}$.

Lescano A.G., Zunt J. 2013: Neuroparasitology and tropical neurology. In: H.H. Garcia, H.B. Tanowitz and O.H. Del Brutto (Eds.), Handbook of Clinical Neurology. Vol. 114, Chapter 27. Elsevier, Amsterdam, pp. 335-345.

Loos-Frank B. 2000: An up-date of Verster's (1969) taxonomic revision of the genus Taenia Linnaeus (Cestoda) in table format. Syst. Parasitol. 45: 155-183.
Maravilla P., Avila G., Cabrera V., Aguilar L., Flisser A. 1998: Comparative development of Taenia solium in experimental models. J. Parasitol. 84: 882-886.

Norma Oficial Mexicana NOM-062-ZOO-1999. 2000. Especificaciones Técnicas para la Producción, Cuidado y Uso de los Animales de Laboratorio. Diario Oficial de México, 59 pp.

Pospekhova N.A., Bondarenko S.K. 2014: Morpho-functional characteristics of the scolex of Wardium chaunense (Cestoda: Aploparaksidae) penetrating into host intestine. Parasitol. Res. 113: 131-137.

Rendón J.L., del Arenal I.P., Guevara-Flores A., MendoZa-Hernández G, Pardo J.P. 2008: Glucose 6-phosphate dehydrogenase from larval Taenia crassiceps (cysticerci): purification and properties. Parasitol. Res. 102: 1351-1357.

Sato H., Kamiya M. 1989: Viable egg production of Taenia crassiceps developed in the intestine of prednisolone-treated golden hamster. Jpn. J. Parastol. 38: 46-53.

Sato H., KamiYa M. 1990: Establishment, development and fecundity of Taenia crassiceps in the intestine of prednisolone-treated Mongolian gerbils and inbred mice. J. Helminthol. 64: 217-222.

Sato H., Kamiya M., Kamiya M. 1994: Infection course of the strobilar stage of Taenia crassiceps in golden hamsters, with reference to host responses. Parasitol. Res. 80: 99-103.

Sato H., OkU Y., Rausch R.L., Kamiya M. 1993: Establishment and survival of the strobilar stage of Taenia crassiceps in hamsters, gerbils and mice, with reference to different helminth isolates. Parasitol. Res. 79: 619-623.

Toral-Bastida E., Garza-Rodriguez A., Jimenez-Gonzalez D.E., Garcia-Cortes R., Avila-Ramirez G., Maravilla P., Flisser A. 2011: Development of Taenia pisiformis in golden hamster (Mesocricetus auratus). Parasit. Vectors 4: 147.

Verster A. 1971: Preliminary report on the golden hamster as a definitive host of Taenia solium Linneus, 1758 and Taenia saginata Goeze, 1782. Onderstepoort J. Vet. Res. 38: 63-64.

Vinaud M.C., Ferreira C.S., Lino R.S. Jr., Bezerra J.B.C. 2008: Taenia crassiceps: energetic and respiratory metabolism from cysticerci exposed to praziquantel and albendazole in vitro. Exp. Parasitol. 120: 221-226.

Willms K., Zurabian R. 2010: Taenia crassiceps: in vivo and in vitro models. Parasitology 137: 335-346.

Zurabian R., Aguilar L., Jiménez J.A., Robert L., Willms K. 2008: Evagination and infectivity of Taenia crassiceps cysticerci in experimental animals. J. Parasitol. 94: 1-6.

Cite this article as: Aguilar-Vega L., Plett-Torres T., Romano M.C. Zurabian R. 2020: Evagination of metacestodes of the WFU strain of Taenia crassiceps and evaluation of the impact of immune suppression of hamsters during tapeworm development. Folia Parasitol. 67: 022. 